\title{
The mass spectrum of four-dimensional modes in seven-dimensional Kaluza-Klein theory with a spontaneous compactification solution of Freund- Rubin type
}

\author{
Sergey Keyzerov ${ }^{1}$ \\ Skobeltsyn Institute of Nuclear Physics, M.V. Lomonosov Moscow State University \\ 1(2), Leninskie gory, GSP-1, Moscow 119991, Russian Federation \\ E-mail: errar@www-hep.sinp.msu.ru
}

\section{Edward Rakhmetov}

Skobeltsyn Institute of Nuclear Physics, M.V. Lomonosov Moscow State University

1(2), Leninskie gory, GSP-1, Moscow 119991, Russian Federation

E-mail:dsparel@mail.ru

A theory of interacting gravity, vector and antisymmetric third-rank tensor field with a spontaneous compactification solution of Freund-Rubin type in seven-dimensional space-time is presented. A class of models is studied, which admit Freund-Rubin type of compactification and, at the same time, possess nontrivial solutions for the vector field. We obtain the mass spectrum of fourdimensional modes, which appears after dimensional reduction. We also demonstrate the absence of tachyon modes and the presence of additional massless vector and scalar modes.

The XXth International Workshop High Energy Physics and Quantum Field Theory

Sochi, Russia

September 24-October 1, 2011

$1 \quad$ Speaker 


\section{Introduction}

Theories with extra spatial dimensions are very popular in searches of new physics nowadays. But there is a well known problem of inconsistent of mass and charge scales. If the extra dimensions are compactified into a manifold with the scale $r$ of the Planck order, then we usually have the realistic charges (of the order about 1 in dimensionless units) but the typical masses (having order close to $r^{-1}$ ) are close to the Plank scale also [1].

Nevertheless, theories with extra dimensions of the Planck order have some mechanisms such that we are able to obtain realistic charges and masses. But such mechanisms require special approaches [2]. In this paper we discuss one of such approaches. For the set of sevendimensional fields mentioned below we perform dimensional reduction and obtain the mass spectrum of four-dimensional modes. We also demonstrate the absence of tachyon modes (when certain relations between theory constants are valid) and the presence of additional massless vector and scalar modes.

\section{Action of the theory and self-consistent spontaneous compactification solution.}

In this section large Latin indices take the values from 0 to 7 , with the exception of 4 , small Latin indices run from 0 to 3 and small Greek indices run from 5 to 7 . The summation over the repeated indices is implied. The action of the theory is

$S=k_{1} \int_{X_{7}} L \sqrt{|G|} d x, L=R-k_{2}-\frac{1}{4} \tilde{F}_{A B} \tilde{F}^{A B}-\frac{k_{3}}{2} \tilde{V}_{A} \tilde{V}^{A}-\frac{1}{48} P_{A B C D} P^{A B C D}$

where $\tilde{F}_{A B} \equiv F_{A B}+k_{4} \alpha_{A B C} V^{C}, \tilde{V}_{A} \equiv V_{A}+k_{5} \alpha_{A B C} F^{B C}$

and $F_{A B} \equiv \partial_{[A} V_{B]}, P_{A B C D} \equiv \partial_{[A} Q_{B C D]}, \alpha^{A B C} \equiv-\frac{1}{24} \varepsilon^{A B C D E F G} P_{D E F G}$

This set of equations has a self-consistent spontaneous compactification solution of the form $A d S_{4}{ }^{\mathrm{x}} \mathrm{S}^{3}$. The ansatz for the metric looks as follows:

$G_{\alpha \beta}\left(x^{\mu}, x^{q}\right) \equiv \eta_{\alpha \beta}\left(x^{\mu}\right), G_{a b}\left(x^{\mu}, x^{q}\right) \equiv g_{a b}\left(x^{q}\right)$

where $g_{a b}\left(x^{q}\right)$ and is the metric tensor on 3-dimensional sphere $\mathrm{S}^{3}$

and $\eta_{\alpha \beta}\left(x^{\mu}\right)$ is the metric tensor of

For the Ricci tensor and for the curvature we obtain

$R_{\alpha \beta}=\frac{K}{4} \eta_{\alpha \beta}, R_{a b}=2 r^{-2} g_{a b}, R=K+6 r^{-2}$

where $r$ is the curvature on 3-dimensional sphere $\mathrm{S}^{3}$ (radius of the sphere) and $K$ is the curvature of

For the vector field $V_{A}$ we have:

$$
\begin{aligned}
& V(a) \equiv \text { const }, V_{a}=g(a)_{a} V(a) \\
& \nabla_{q} V^{q}=\nabla(a) V(a)=\partial(a) V(a)=0, \nabla_{a} V_{b}=r^{-1} \varepsilon_{a b c} V^{c} \\
& F(a b)=2 r^{-1} \varepsilon(a b c) V(c), F_{a b}=\nabla_{[a} V_{b]}=2 r^{-1} \varepsilon_{a b c} V^{c}
\end{aligned}
$$


where $g(a)_{q}$ is a left-invariant frame on 3-dimensional sphere $S^{3}$ such that

$[\partial(a), \partial(b)]=2 r^{-1} \varepsilon(a b c) \partial(c)$

Here indices in the brackets are frame (vielbein) indices, which run from 5 to 7 , and the summation over the repeated indices is implied.

For the antisymmetric third-rank tensor field $Q_{B C D}$ we obtain:

$Q_{\alpha \beta \gamma}=\varepsilon_{\alpha \beta \gamma \mu} Q^{\mu}$

$P_{\alpha \beta \gamma \delta}=\varepsilon_{\alpha \beta \gamma \delta} \alpha=-6 \varepsilon_{\alpha \beta \gamma \delta} \nabla_{\mu} Q^{\mu}, \Rightarrow \alpha(a b c)=\varepsilon(a b c) \alpha$ and $\alpha \equiv$ const

All the other components of all fields and their stress tensors are trivial (are equal to zero).

If we take values of the constants, such that

$\alpha^{2}=\frac{4 k_{2}-5 K}{4}, r^{-2}=\frac{4 k_{2}-3 K}{16}, k_{4} \alpha=-2 r^{-1}, 4 r^{-1} k_{5} \alpha=-1$

then we get a solution for the metric tensor corresponding to the desired direct product structure $\mathrm{M}_{4}{ }^{\mathrm{x}} \mathrm{S}^{3}$ and solutions for vector and antisymmetric third-rank tensor fields of the form (2.6)-(2.11) mentioned above [3]. That is to say, such values of the theory constants allow Freund-Rubin type of compactification (with anti de Sitter space and the other dimensions compactified into 3-dimensional sphere) and, at the same time, admit nontrivial solutions for the vector field [3].

\section{Dimensional reduction and mass spectrum of four-dimensional modes}

\subsection{Equations for the small field fluctuation above the Freund-Rubin background}

7-dimentional equations for the small field fluctuation above the Freund-Rubin background can be obtained from the equations of motion [3] for such systems with the action (2.1)-(2.3):

$$
\begin{aligned}
& \mathbf{x}_{A B} \equiv \delta R_{A B}-2 r^{-2} \delta G_{A B}+ \\
& +\frac{1}{2} G_{A B}\left\{-\delta R+\frac{\alpha}{24} \varepsilon^{\mu \nu \lambda \tau} \delta P_{\mu \nu \lambda \tau}+\frac{\alpha^{2}}{2} \eta^{\mu \nu} \delta G_{\mu \nu}+r^{-1} V_{l} \varepsilon^{l p q} \delta \tilde{F}_{p q}+k_{3} V^{l} \delta \tilde{V}_{l}\right\}+ \\
& +r^{-1} V_{l} \varepsilon_{(A}^{l q} \delta \tilde{F}_{B) q}-\frac{k_{3}}{2} V_{(A} \delta \tilde{V}_{B)}-\frac{1}{12} P_{(A}^{P Q R} \delta P_{B) P Q R}+\frac{\alpha^{2}}{2}\left(\delta_{A}^{\mu} \delta_{B}^{v}-\eta_{A B} \eta^{\mu \nu}\right) \delta G_{\mu \nu}=0 \\
& \mathbf{y}_{A} \equiv r^{-1} \varepsilon_{A}{ }^{p q} \delta \tilde{F}_{p q}-k_{3} \delta \tilde{V}_{A}+\nabla^{P}\left(\delta \tilde{F}_{P A}-\frac{k_{3} r}{2} \varepsilon_{P A}{ }^{q} \delta \tilde{V}_{q}\right)=0 \\
& \mathbf{z}_{A B C} \equiv-\frac{\alpha}{2} \varepsilon_{A B C}^{\mu} G^{X Y} \nabla_{\mu} \delta G_{X Y}+ \\
& +\nabla^{P}\left(-\frac{k_{4}}{2} \varepsilon_{P A B C}{ }^{X Y l} V_{l} \delta \tilde{F}_{X Y}-2 r^{-1} k_{3} k_{5} \varepsilon_{P A B C}{ }^{x y Q} \varepsilon_{x y l} V^{l} \delta \tilde{V}_{Q}+\delta P_{P A B C}-\frac{\alpha}{6} \varepsilon_{[P A B}{ }^{v} \delta G_{C] v}\right)=0
\end{aligned}
$$

For the sake of brevity we consider a 3 -index antisymmetric tensor such that $\varepsilon_{A B C}$ is equal to zero if one of its index has 4-dimensional value, and $\varepsilon_{A B C}$ is the Levi-Chivita tensor on 3dimensional sphere if all its indices corresponds with additional coordinates. 


\subsection{Dimensional reduction}

To obtain an effective 4-dimentional theory, it is necessary to expand all the fields of the 7-dimentional theory into series of full sets of functions, depending on additional coordinates.

Suppose we use sets of eigen functions of the Laplass, Maxwell and Lichnerowicz operators on 3-dimentional sphere for the scalar, vector and tensor fields of the 7-dimentional theory correspondingly. By definition, we have for these functions

$$
\begin{aligned}
& \nabla_{q} \nabla^{q} \Omega_{\mathbf{J}} \equiv \Delta \Omega_{\mathbf{J}}=-r^{-2} \Delta_{\mathbf{J}} \Omega_{\mathbf{J}}, \Delta_{\mathbf{J}} \equiv n(n+2) \\
& \nabla^{q} \nabla_{[q} \Omega_{a] \mathbf{K}}=-r^{-2} \lambda_{\mathbf{K}} \Omega_{a \mathbf{K}} \\
& \nabla_{q} \nabla_{(a} \Omega_{b) \mathbf{L}}^{q}-\nabla_{a} \nabla_{b} \Omega_{q \mathbf{L}}^{q}-\nabla_{q} \nabla^{q} \Omega_{a b \mathbf{L}}-g_{a b} \nabla_{q} \nabla^{[p} \Omega_{p \mathbf{L}}^{q]}-4 r^{-2} \Omega_{a b \mathbf{L}}+2 r^{-2} g_{a b} \Omega_{q \mathbf{L}}^{q}= \\
& =-r^{-2} \kappa_{\mathbf{L}}\left(\Omega_{a b \mathbf{L}}-g_{a b} \Omega_{q \mathbf{L}}^{q}\right)
\end{aligned}
$$

These functions are characterized by three numbers $(n, m, l)$ such that

$n=0,1,2, \ldots, m=-n,-n+2, \ldots, n$ and $l=-n,-n+2, \ldots, n$

Furthermore, for Maxwell and Lichnerowicz eigen functions some relations are valid

$$
\begin{aligned}
& \nabla^{a} \Omega_{a \mathbf{K}}=0, \nabla_{q} \Omega_{b \mathbf{L}}^{q}=0, \Omega_{q \mathbf{L}}^{q}=0 \\
& \nabla_{[a} \Omega_{b] \mathbf{K}}=r^{-1} \sqrt{\lambda_{\mathbf{K}}} \varepsilon_{a b c} \Omega^{c}{ }_{\mathbf{K}}
\end{aligned}
$$

The expansions for the small field fluctuation are given by

$$
\begin{aligned}
& \delta G_{\alpha \beta}=\sum_{\mathbf{J}} h_{\alpha \beta}{ }^{\mathbf{J}} \Omega_{\mathbf{J}} \\
& \delta G_{a \beta}=\sum_{\mathbf{K}} h_{\beta}{ }^{\mathbf{K}} \Omega_{a \mathbf{K}}+\sum_{\mathbf{J}} a_{\beta}{ }^{\mathbf{J}} \nabla_{a} \Omega_{\mathbf{J}} \\
& \delta G_{a b}=\sum_{\mathbf{L}} h^{\mathbf{L}} \Omega_{a b \mathbf{L}}+\sum_{\mathbf{K}} b^{\mathbf{K}} \nabla_{(a} \Omega_{b) \mathbf{K}}+\sum_{\mathbf{J}}\left\{c^{\mathbf{J}} \nabla_{a} \nabla_{b}+d^{\mathbf{J}} g_{a b}\right\} \Omega_{\mathbf{J}} \\
& \delta V_{\alpha}=\sum_{\mathbf{J}} v_{\alpha}{ }^{\mathbf{J}} \Omega_{\mathbf{J}} \\
& \delta V_{a}=\sum_{\mathbf{K}} v^{\mathbf{K}} \Omega_{a \mathbf{K}}+\sum_{\mathbf{J}} u^{\mathbf{J}} \nabla_{a} \Omega_{\mathbf{J}} \\
& \delta Q_{\alpha \beta \gamma}=\varepsilon_{\alpha \beta \gamma}{ }^{\mu} \sum_{\mathbf{J}} q_{\mu}{ }^{\mathbf{J}} \Omega_{\mathbf{J}} \\
& \delta Q_{a \beta \gamma}=\sum_{\mathbf{K}} q_{\beta \gamma}{ }^{\mathbf{K}} \Omega_{a \mathbf{K}}+\sum_{\mathbf{J}} f_{\beta \gamma}{ }^{\mathbf{J}} \nabla_{a} \Omega_{\mathbf{J}} \\
& \delta Q_{a b \gamma}=\varepsilon_{a b}{ }^{q}\left\{\sum_{\mathbf{K}} u_{\gamma}{ }^{\mathbf{K}} \Omega_{q \mathbf{K}}+\sum_{\mathbf{J}} w_{\gamma}{ }^{\mathbf{J}} \nabla_{q} \Omega_{\mathbf{J}}\right\} \\
& \delta Q_{a b c}=\varepsilon_{a b c} \sum_{\mathbf{J}} q^{\mathbf{J}} \Omega_{\mathbf{J}}
\end{aligned}
$$

where the fields $h_{\alpha \beta}{ }^{\mathbf{J}}, h_{\beta}{ }^{\mathbf{K}}, a_{\beta}{ }^{\mathbf{J}}, h^{\mathbf{L}}, b^{\mathbf{K}}, c^{\mathbf{J}}, d^{\mathbf{J}}, v_{\alpha}{ }^{\mathbf{J}}, v^{\mathbf{K}}, u^{\mathbf{J}}, q_{\mu}{ }^{\mathbf{J}}, q_{\beta \gamma}{ }^{\mathbf{K}}, f_{\beta \gamma}{ }^{\mathbf{J}}, u_{\gamma}{ }^{\mathbf{K}}, w_{\gamma}{ }^{\mathbf{J}}, q^{\mathbf{J}}$ depend on 4-dimetional coordinates $x^{\mu}$ only. Antisymmetric second-rank tensor fields $q_{\beta \gamma}{ }^{\mathbf{K}} f_{\beta \gamma}{ }^{\mathbf{J}}$ can be expressed as

$$
q_{\beta \gamma}{ }^{\mathbf{K}} \equiv \nabla_{[\beta} \sigma_{\gamma]}{ }^{\mathbf{K}}+\varepsilon_{\beta \gamma}{ }^{\mu \nu} \nabla_{\mu} \omega_{v}{ }^{\mathbf{K}}, f_{\beta \gamma}{ }^{\mathbf{J}} \equiv \nabla_{[\beta} \rho_{\gamma]}{ }^{\mathbf{J}}+\varepsilon_{\beta \gamma}{ }^{\mu v} \nabla_{\mu} \theta_{v}{ }^{\mathbf{J}}
$$

Hence, after dimensional reduction we have the effective 4-dimentional theory with sets of scalar, vector and symmetric second-rank tensor fields.

Substituting expansions (3.9) for the small field fluctuation in (3.1)-(3.3), we get $\mathbf{x}_{\alpha \beta} \equiv \sum_{\mathbf{J}} \mathbf{x}_{\alpha \beta}{ }^{\mathbf{J}} \Omega_{\mathbf{J}}=0$ 


$$
\begin{aligned}
& \mathbf{x}_{a \beta} \equiv \sum_{\mathbf{K}} \mathbf{x}_{\mathrm{I} \beta}{ }^{\mathbf{K}} \Omega_{a \mathbf{K}}+\sum_{\mathbf{J}} \mathbf{x}_{\mathrm{II} \beta}{ }^{\mathbf{J}} \nabla_{a} \Omega_{\mathbf{J}}=0 \\
& \mathbf{x}_{a b} \equiv \sum_{\mathbf{L}} \mathbf{x}_{\mathrm{I}}{ }^{\mathbf{L}} \Omega_{a b \mathbf{L}}+\sum_{\mathbf{K}} \mathbf{x}_{\mathrm{II}}{ }^{\mathbf{K}} \nabla_{(a} \Omega_{b) \mathbf{K}}+\sum_{\mathbf{J}}\left\{\mathbf{x}_{\mathrm{III}}{ }^{\mathbf{J}} \nabla_{a} \nabla_{b}+\mathbf{x}_{\mathrm{IV}}{ }^{\mathbf{J}} g_{a b}\right\} \Omega_{\mathbf{J}}=0
\end{aligned}
$$

This is equivalent to the set of 4-dimentional equations:

$\mathbf{x}_{\alpha \beta}{ }^{\mathbf{J}}=0$

$\mathbf{x}_{\mathrm{I} \beta}{ }^{\mathrm{K}}=0, \mathbf{x}_{\mathrm{II} \beta}{ }^{\mathrm{J}}=0$

$\mathbf{x}_{\mathrm{I}}^{\mathbf{L}}=0, \quad \mathbf{x}_{\mathrm{II}}^{\mathbf{K}}=0, \mathbf{x}_{\mathrm{III}}^{\mathbf{J}}=0, \mathbf{x}_{\mathrm{IV}}{ }^{\mathbf{J}}=0$

For example, let us write down the detailed expression for $\mathbf{x}_{\alpha \beta}{ }^{\mathbf{J}}=0$ (For the sake of brevity the curvature $K$ is equal to zero)

$$
\begin{aligned}
& \mathbf{x}_{\alpha \beta}{ }^{J}=\frac{1}{2}\left\{\nabla_{\mu} \nabla_{(\alpha} h_{\beta)}^{\mu J}-\nabla_{\alpha} \nabla_{\beta} h_{\mu}^{\mu J}-\nabla_{\mu} \nabla^{\mu} h_{\alpha \beta}{ }^{J}-\eta_{\alpha \beta} \nabla_{\mu} \nabla^{[v} h_{v}^{\mu] J}-r^{-2} \Delta_{\mathbf{J}}\left(\eta_{\alpha \beta} h_{\mu}^{\mu J}-h_{\alpha \beta}{ }^{J}\right)\right\}+ \\
& -\frac{1}{2} r^{-2} \Delta_{\mathbf{J}}\left(\nabla_{(\alpha} a_{\beta)}{ }^{J}-2 \eta_{\alpha \beta} \nabla_{\mu} a^{\mu J}\right)+\frac{1}{2}\left\{\left(\eta_{\alpha \beta} \square-\nabla_{\alpha} \nabla_{\beta}\right)\left(-r^{-2} \Delta_{\mathbf{J}} c^{\mathbf{J}}+3 d^{\mathbf{J}}\right)+\right. \\
& \left.+r^{-2} \eta_{\alpha \beta}\left[2 \Delta_{\mathbf{J}}\left(r^{-2} c^{\mathbf{J}}-d^{\mathbf{J}}\right)+\kappa V^{2}\left(-r^{-2} \Delta_{\mathbf{J}} c^{\mathbf{J}}+3 d^{\mathbf{J}}\right)+(4+\kappa) V^{2} \chi_{\mathbf{L}}^{\mathbf{J}} h_{p q}{ }^{\mathbf{L}}\right]\right\}+ \\
& +\frac{1}{2} r^{-2} \eta_{\alpha \beta}\left\{\frac{1}{2}\left(4-(4+\kappa) V^{2}\right) \varphi^{\mathbf{J}}+\frac{1}{2}(4-\kappa)|V|\left[\left(\sqrt{\lambda_{\mathbf{K}}}-2\right) \zeta_{\mathbf{K}} v^{\mathbf{J}}-2 m_{\mathbf{J}} u^{\mathbf{J}}\right]\right\}=0
\end{aligned}
$$

where $V^{q} \Omega_{q \mathbf{K}} \equiv|V| \zeta_{\mathbf{K}}^{\mathbf{J}} \Omega_{\mathbf{J}}, V^{p} V^{q} \Omega_{p q \mathbf{L}} \equiv V^{2} \chi_{\mathbf{L}}^{\mathbf{J}} \Omega_{\mathbf{J}}, V^{p} \Omega_{p q \mathbf{L}} \equiv|V| \xi_{\mathbf{L}}^{\mathbf{K}} \Omega_{q \mathbf{K}}$

and $\varphi^{\mathrm{J}} \equiv-\frac{12}{\alpha} \nabla_{\mu} q^{\mu \mathrm{J}}-h_{\mu}^{\mu \mathrm{J}}-r^{-2} \Delta_{\mathbf{J}} c^{\mathbf{J}}+3 d^{\mathrm{J}}$

For the sake of brevity all the other equations (3.12) can be written as follows

$\mathbf{A}_{J}^{I} \nabla^{\mu} \nabla_{[\mu} \Psi_{\beta]}^{J}+r^{-2} \mathbf{B}_{J}^{I} \Psi_{\beta}^{J}=0$

$\mathbf{C}_{J}^{I} \square U^{J}+r^{-2} \mathbf{D}_{J}^{I} U^{J}=0$

where $\Psi_{\beta}^{J} \equiv\left\{h_{\beta}{ }^{\mathbf{K}}, u_{\gamma}{ }^{\mathbf{K}}, \sigma_{\gamma}{ }^{\mathbf{K}}, \omega_{\gamma}{ }^{\mathbf{K}}, a_{\beta}{ }^{\mathbf{J}}, v_{\alpha}{ }^{\mathbf{J}}, q_{\mu}{ }^{\mathbf{J}}, w_{\gamma}{ }^{\mathbf{J}}, \rho_{\gamma}{ }^{\mathbf{J}}, \theta_{v}{ }^{\mathrm{J}}\right\}$ is a column consructed from vector fields, $U^{J} \equiv\left\{h^{\mathrm{L}}, b^{\mathrm{K}}, v^{\mathrm{K}}, c^{\mathrm{J}}, d^{\mathrm{J}}, u^{\mathrm{J}}, q^{\mathrm{J}}\right\}$ is a column of scalar field. $\mathbf{A}_{J}^{I}, \mathbf{B}_{J}^{I}, \mathbf{C}_{J}^{I}, \mathbf{D}_{J}^{I}$ are nfinitedimensional block-diagonal matrices.

\subsection{Mass spectrum of four-dimensional modes}

For example, let us consider the vector field system (3.16). The number of equations in the system (3.16) doesn't correspond to the number of vector fields and some equations are algebraic. These algebraic equations are just equations of constraints, and allow us to exclude some fields from the system. The general purpose is to reduce the system (3.16) to a new system with a less number of pure dynamic equations

$\nabla^{\mu} \nabla_{[\mu} \Psi_{\beta]}^{i}+r^{-2} \mathbf{M}_{j}^{i} \Psi_{\beta}^{j}=0$

where $\mathbf{M}_{j}^{i}$ - is a diagonal matrix. Obviously, the eigenvalues $r^{-2} \lambda$ of the matrix $r^{-2} \mathbf{M}_{j}^{i}$ are squared masses of the vector fields.

Loosing some details, we'll list diagonal elements $\lambda$ of this matrix $\mathbf{M}_{j}^{i}$ for all 4dimentional modes. As far as all these equations are linear, the expansion in eigen functions of the Laplass, Maxwell and Lichnerowicz operators separate modes with the same number n, i.e. 
system is valid for every n separately. Suppose $\kappa \equiv k_{3} r^{2}$; then we have the eigenvalues $\lambda$ of the matrix $\mathbf{M}_{j}^{i}$ for

\section{Vector sector:}

1. $\mathrm{n}=0$

$\lambda=0$

(1 singlet state)

$\lambda=\frac{2}{4-\kappa V^{2}}\left\{8+\kappa\left(1+V^{2}\right) \pm \sqrt{\left[8+\kappa\left(1+V^{2}\right)\right]^{2}-8 \kappa\left(4-\kappa V^{2}\right)\left(1+V^{2}\right)}\right\}$

(2 singlet states)

$\lambda=\frac{2}{1-V^{2}}\left\{2-V^{2} \pm \sqrt{\left(2-V^{2}\right)^{2}-4\left(1-V^{2}\right)\left(3+4 V^{2}\right)}\right\}$

(2 singlet states)

There are no tachyon modes if $0<V^{2}<1$ and $\kappa<4 / V^{2}$

2. $\mathrm{n}=2$

$\lambda=0$

(1 triplet state)

$\lambda=\Delta_{0} \equiv n(n+2)=8$

(2 triplet states)

$\lambda=\frac{2\left[32+\kappa\left(1-3 V^{2}\right)\right]}{4-\kappa V^{2}}\left\{1 \pm \sqrt{1-24 \frac{32+4 \kappa\left(1-V^{2}\right)-\kappa^{2} V^{2}\left(1+V^{2}\right)}{\left[32+\kappa\left(1-3 V^{2}\right)\right]^{2}}}\right\}$

(2 triplet states)

$\lambda=8+\frac{m^{2} V^{2}}{1-V^{2}}$

(1 triplet state)

3. $\mathrm{n}>0, \mathrm{n} \neq 2$

$\lambda=\Delta_{0} \equiv n(n+2)$

(2 n-let states)

$\lambda_{i}$

(3 n-let states)

where $\lambda_{i}$ are solutions of cubic equation

$$
\begin{aligned}
& f(\lambda)=\left(4-\kappa V^{2}\right) \lambda^{3}-2\left[16-6 \Delta_{0}+\kappa\left(2+V^{2}+V^{2} \Delta_{0}\right)\right] \lambda^{2}+ \\
& +\left[12 \Delta_{0}^{2}+\kappa\left(32\left(1+V^{2}\right)-\Delta_{0}\left(8+16 V^{2}+V^{2} \Delta_{0}\right)\right)\right] \lambda+4 \Delta_{0}\left(\Delta_{0}+8\right)\left[\Delta_{0}-\kappa\left(1+V^{2}\right)\right]=0
\end{aligned}
$$

We see that there are no tachyon modes in vector sector if $0<V^{2}<1$ and $\kappa<4 / V^{2}$. In addition, in vector sector we have four massless modes (one singlet and one triplet state). All the other modes have positive squared masses of the Planck order (of the order $r^{-2}$ ).

Scalar sector:

1. $\mathrm{n}=0$

$\lambda=0$

(2 singlet states)

$\lambda=8$

(3 singlet states)

$\lambda=8+(4+\kappa) V^{2}$

(2 singlet states)

2. $\mathrm{n}=2$

$\lambda=\frac{1}{4-\kappa V^{2}}\left\{4+\kappa\left(1+V^{2}\right) \pm \sqrt{\left[4+\kappa\left(1+V^{2}\right)\right]^{2}-\kappa\left(4-\kappa V^{2}\right)\left(1-V^{2}\right)}\right\}$

(2 triplet states)

3. $\mathrm{n}>0, \mathrm{n} \neq 2$

$\lambda=\Delta_{0} \equiv n(n+2)$

(1 n-let states) 
$\lambda_{i}$

where $\lambda_{i}$ are solutions of cubic equation

$$
\begin{aligned}
& f(\lambda)=\left(4-\kappa\left(1-V^{2}\right)\right) \lambda^{3}-2\left[16+\kappa\left(1+V^{2}\right)\right] \lambda^{2}+ \\
& +8\left[2-\kappa\left(1+2 V^{2}\right)\right] \Delta_{0} \lambda+4 \Delta_{0}\left(\Delta_{0}+8\right)\left[\Delta_{0}+1-V^{2}\right]=0
\end{aligned}
$$

As we can see there are no tachyon modes in scalar sector if $0<V^{2}<1$ and $\kappa<4 / V^{2}$. In addition, in scalar sector we have two massless singlet states. All the other modes have positive squared masses of the Planck order (of the order $r^{-2}$ )

\section{Conclusions}

It was shown in this paper that the model with interacting gravity, vector and antisymmetric third-rank tensor field has some interesting properties:

1. It has a spontaneous compactification solution of Freund-Rubin type in sevendimensional space-time such that the metric has direct product structure $A d S_{4}{ }^{\mathrm{x}} \mathrm{S}^{3}$

2. This spontaneous compactification solution is stable (there are no four-dimensional tachyon modes), when certain relations between theory constants are valid.

3. After dimensional reduction we obtain additional massless vector and scalar fourdimensional modes (one singlet vestor state, one triplet vector state and two scalar singlet states).

4. The model possesses a nontrivial background solution for the vector field such that it depends on additional coordinates only. Such property seems to be useful to obtain leftright asymmetry solutions for models with fermion fields [4].

We suppose that such model will be useful for constructing theories with realistic charges and masses and with axial currents. Such currents arise as a result of interaction between sevendimensional fermions and aforementioned nontrivial background solution for the vector field.

\section{Acknowledgements}

The authors are grateful to E.E. Boos, I.P. Volobuev and M.N. Smolyakov for valuable discussions. The work was supported by FASI state contract 02.740 .11 .0244 and by grant of Russian Ministry of Education and Science NS-4142.2010.2.

\section{References}

[1] A. Salam, J. Strathdee On Kaluza-Klein Theory, Annals of Physics 141 (316) 1982.

[2] I.P. Volobuev, Yu.A. Kubyshin, Differential geometry, Lee algebra and their applications in field theory, Moscow, URSS 1998.

[3] E.R. Rakhmetov, S.I. Keizerov A class of model with a spontaneous compactification of FreundRubin type and nontrivial solution for the vector field in seven-dimensional space-time (accepted in Moscow University Physics Bulletin) 2012.

[4] W. Thirring Acta Phys. Austriaca Suppl. 9 (256-271) 1972 